\title{
HHLA2 Expression is Associated with Poor Survival in Patients with Hepatocellular Carcinoma
}

\author{
Yituo $\mathrm{Xu}$ \\ Zhijie Huang' \\ Xingjuan $\mathrm{Yu}^{\mathrm{I}}$ \\ Zhixiong $\mathrm{Li}^{\mathrm{I}}$ \\ Limin Zheng ${ }^{1,2}$ \\ Jing $X u^{\prime}$ \\ 'Collaborative Innovation Center for \\ Cancer Medicine, State Key Laboratory \\ of Oncology in South China, Sun Yat-sen \\ University Cancer Center, Guangzhou, \\ 510060, People's Republic of China; \\ ${ }^{2}$ MOE Key Laboratory of Gene Function \\ and Regulation, State Key Laboratory of \\ Biocontrol, School of Life Sciences, Sun \\ Yat-sen University, Guangzhou, 510060, \\ People's Republic of China
}

Correspondence: Jing Xu; Limin Zheng Collaborative Innovation Center for Cancer Medicine, State Key Laboratory of Oncology in South China, Sun Yat-sen University Cancer Center, Guangzhou, 510060, People's Republic of China Email xujing@sysucc.org.cn; zhenglm@mail.sysu.edu.cn
Background: Human endogenous retrovirus-H long terminal repeat-associating protein 2 (HHLA2) is a member of the B7 family; however, little is known regarding its expression and clinical relevance in hepatocellular carcinoma (HCC).

Methods: To better characterize HHLA2 expression in HCC, we analyzed its expression by in situ staining and further investigated its correlation with immune infiltration and patient prognosis.

Results: HHLA2 was primarily expressed in the peri-tumor region of HCC tissues and colocalized with $\mathrm{CD} 8^{+}$monocytes/macrophages. In vitro analysis and multiimmunofluorescence staining showed up-regulated HHLA2 expression in tumor-activated monocytes/macrophages, and HHLA2 ${ }^{+}$monocytes/macrophages expressed high levels of HLA-DR in HCC tissue. A correlation analysis showed that samples displaying high HHLA2 expression in the peri-tumor region had significant tumor infiltration of CD204 and $\mathrm{CD}_{11 \mathrm{~b}^{+}}$cells, and low expression of genes associated with an anti-tumor immune response. The high level of peri-tumoral HHLA2 expression was associated with a poor patient overall survival (OS; $P=0.008$ ). A multivariate analysis revealed that HHLA2 expression in the peri-tumor region was an independent prognostic factor for OS (hazard ratio $=1.872, p=0.003)$. Moreover, the expression of HHLA2 was negatively correlated with PD-L1, and patients exhibiting HHLA2 and programmed cell death-ligand 1(PD-L1) co-expression had the shortest survival time.

Conclusion: HHLA2 expression represented an immunosuppressive microenvironment in $\mathrm{HCC}$, and may serve as a potential target for immunotherapy.

Keywords: HHLA2, monocytes/macrophages, HCC, prognosis

\section{Introduction}

Hepatocellular carcinoma (HCC) is among the most prevalent tumors, and is associated with increasing mortality worldwide. ${ }^{1,2}$ Due to the lack of biological indicators in the early stages, most HCC patients are diagnosed as middle-advanced at the time of treatment. Although surgical intervention represents the most effective therapy for HCC, overall survival remains poor. Immunotherapy using immune checkpoint blockers (ICB) has shown great promise in different types of cancer, including $\mathrm{HCC}$; however, the response rate remains limited. ${ }^{3}$ A central theme in cancer treatment is determining methods of improving and widening the clinical benefit of ICB therapy.

Immune checkpoints refer to a large number of co-stimulatory and co-inhibitory pathways inherent in the immune system that are essential for maintaining selftolerance and regulating the duration and intensity of the physiological immune 
response of peripheral tissues. ${ }^{4}$ Under normal circumstances, immune checkpoints can respond to infections and protect the body from damage; however, immune checkpoint expression often leads to an imbalance of anti-tumor immune function in malignant tumors, thereby promoting tumor growth. ${ }^{5,6}$ Members of the B7-CD28 pathways represent the most widely studied immune checkpoint molecules, which can be divided into three groups according to the following phylogenetic tree: 1) CD80/CD86/CD28/cytotoxic T-lymphocyte associated protein 4 (CTLA4), which primarily regulates central immune tolerance and is critical for regulating the early stages of $\mathrm{T}$ cell responses; 2) PD-L1 /programmed cell death-ligand 2 (PD-L2)/ programmed cell death protein 1 (PD-1); and 3) B7-H3/B7-H4/HHLA2/ $\mathrm{CD} 28 \mathrm{H}$. The last two groups of molecules mainly regulate peripheral immunity and lead to immune tolerance in the tumor microenvironment by inhibiting $\mathrm{T}$ cell proliferation and function. ${ }^{6}$ Currently, the majority of immune checkpoint blockers have been targeted towards CTLA4 and PD-1/PDL1. ${ }^{7}$

Human endogenous retrovirus-H long terminal repeat-associating protein 2 (HHLA2, also known as B7-H7), named due to the presence of polyadenylated repeating at the long end of the 3 '-untranslated region sequence, and is analogous to $\mathrm{B} 7-\mathrm{H} 3$ and $\mathrm{B} 7-\mathrm{H} 4 .^{8,9} \mathrm{In}$ normal tissues, HHLA2 is expressed in the trophoblast cells of placenta, as well as the epithelial cells of the intestine, kidney, gallbladder, and breast. ${ }^{10}$ While human monocytes and B cells can also express HHLA2 when induced by lipopolysaccharide (LPS) or Interferon gamma (IFN- $\gamma$ ) inflammation, the function of HHLA2 on $\mathrm{T}$ cells remains controversial. ${ }^{11-14}$ HHLA2 has been reported to be expressed on tumor cells of various cancers, including human pancreatic ductal adenocarcinoma, intrahepatic cholangiocarcinoma, and clear cell renal cell carcinoma. ${ }^{10,15-19}$ The high level of HHLA2 expression is correlated with increased tumor size and advanced TNM stage, indicating its essential role in tumor development. HHLA2 expression on tumor infiltrating-immune cells has not yet been reported, and its role in the tumor immune microenvironment of HCC is not completely understood.

In this study, we examined the level of HHLA2 expression in HCC tissues, and found that HHLA2 was primarily expressed on stromal cells and co-localized with $\mathrm{CD}^{+} 8^{+}$ monocytes/macrophages. High HHLA2 expression was associated with an immunosuppressive microenvironment, and correlated with worse clinical outcomes of HCC patients. In addition, the pattern of HHLA2 and PD-L1 expression was further explored.

\section{Materials and Methods Patients}

A total of 205 HCC patients who had received a tumor resection at the Sun Yat-sen University Cancer Center between 2006 and 2010 were enrolled in this study. ${ }^{20,21}$ The inclusion criteria were as follows: no concurrent autoimmune disease, human immunodeficiency virus (HIV), or syphilis, as well as an absence of anticancer therapy or distant metastasis before surgery. All patients underwent an HCC curative resection with the following intraoperative aims: At least $1 \mathrm{~cm}$ in a resection margin, all tumor nodules were completely resected, and the cut surface free of tumor left (based on histological examination). Intra-operative ultrasound and post-surgical contrastenhanced computed tomography were applied to ensure that the HCC was completely removed. Relapse-free survival (RFS) was defined as the interval between surgery and the first of recurrence or death, or between surgery and the final observation for patients without recurrence. Overall survival (OS) was defined as the interval between surgery and death or between surgery and the last observation for surviving patients.

All samples were anonymously coded in accordance with local ethical guidelines (in accordance with the Declaration of Helsinki). Written informed consent was obtained and the study protocol was approved by the Review Board of Sun Yat-sen University Cancer Center, Guangzhou, China.

\section{Antibodies}

The following primary antibodies were used in this study: mouse anti-human CD68 monoclonal antibody (Dako, Carpinteria, CA, USA), rabbit anti-human PD-L1 monoclonal antibody (Cell Signaling Technology, Danvers, MA, USA), rabbit anti-human HHLA2 polyclonal antibody (Cell Signaling Technology, Danvers, MA, USA), rabbit anti-human CD11b monoclonal antibody (Abcam, Cambridge, MA, USA), mouse anti-human CD204 monoclonal antibody (Transgenic, Kumamoto, Japan), mouse anti-human CD163 monoclonal antibody (ZSBio, Beijing, China), and mouse anti-human CD15 monoclonal antibody (ZSBio, Beijing, China). 


\section{Immunohistochemistry (IHC)}

Formalin-fixed, paraffin-embedded hepatocellular carcinoma tissues were sliced into $4-\mu \mathrm{m}$ thick sections to be stained for IHC. The sections were dewaxed in xylene and rehydrated through a decreasing gradient of ethanol. The sections were placed in $0.3 \% \mathrm{H}_{2} \mathrm{O}_{2}$ for $10 \mathrm{~min}$ at room temperature to reduce endogenous peroxidase activity. Heat-mediated retrieval was used for antigen retrieval. The slides were incubated with different primary antibodies and incubated overnight at $4^{\circ} \mathrm{C}$. Detection was performed using an Envision Detection Systems (DakoCytomation, Carpinteria, CA, USA) in accordance with the manufacturer's instructions. The sections were then counterstained with hematoxylin. Images were captured using an Eclipse advanced research microscope (Nikon, Melville, NY, USA).

\section{Multiplex Staining}

Tyramide signal amplification (TSA) Plus Fluorescence Kits (PerkinElmer, Foster City, CA, USA) combined with IHC (TSA-IHC) were used for multiplex immunofluorescence staining. ${ }^{20}$ Briefly, different primary antibodies were sequentially applied, followed by an incubation with horseradish peroxidase-conjugated secondary antibody and tyramine signal amplification. Nuclei were stained with 4'-6'-diamidino-2-phenylindole (DAPI, Thermo Scientific, Waltham, Massachusetts, USA) after labelling of all the human antigens. For the colocalization analysis, images were acquired with a laser confocal microscope (Olympus, Essex, UK) and analyzed with FV10-ASW Viewer software (Olympus, Essex, UK).

\section{Isolation of Mononuclear Cells from the Peripheral Blood}

Ficoll density gradient centrifugation was applied to isolate peripheral blood mononuclear cells (PBMCs). CD14 monocytes were isolated using magnetic beads (Miltenyi Biotec, Auburn, CA, USA) in accordance with the manufacturer's protocol. Monocytes in Dulbecco's Modified Eagle Medium (DMEM) (Thermo Scientific, Waltham, Massachusetts, USA) alone were plated at a density of 5 $\times 10^{6} /$ well in 24-well plates for $0.5 \mathrm{~h}$, and then cultured in DMEM containing $10 \%$ human $\mathrm{AB}$ serum for $24 \mathrm{~h}$. The monocytes in DMEM containing $\mathrm{AB}$ serum were cultured in the presence of $10 \%$ tumor culture supernatants (TSN) or medium alone for $2 \mathrm{~h}$ to 7 days.

\section{Cell Line and Preparation of TSN}

SK-Hep-1 cell lines were obtained from American Type Culture Collection, and were tested for mycoplasma contamination using single-step polymerase chain reaction (PCR). The cells were maintained in DMEM supplemented with $10 \%$ FBS.

TSN were prepared as previously described. ${ }^{22}$ Briefly, the cells were cultured in DMEM supplemented with $10 \%$ Fetal Bovine Serum (FBS), $100 \mathrm{U} / \mathrm{mL}$ penicillin (SigmaAldrich, Burlington, MA, USA), and $100 \mu \mathrm{g} / \mathrm{mL}$ streptomycin (Sigma-Aldrich, Burlington, MA, USA) in a humidified $5 \% \mathrm{CO}_{2}$ incubator at $37^{\circ} \mathrm{C}$. TSNs were prepared by plating $5 \times 10^{6}$ tumor cells in $10 \mathrm{~mL}$ complete medium in $10 \mathrm{~cm}$ dishes for $24 \mathrm{~h}$, after which the medium was exchanged with DMEM supplemented with $10 \% \mathrm{AB}$ serum. The cells were cultured for $48 \mathrm{~h}$ under these conditions, and the supernatants was collected, centrifuged, and stored in aliquots at $-80^{\circ} \mathrm{C}$.

\section{Flow Cytometry}

Monocytes were detached with $4 \mathrm{mM}$ EDTA for $1 \mathrm{~h}$, washed, and resuspended in phosphate buffered saline (PBS) supplemented with 1\% heat-inactivated fetal bovine serum. The monocytes were then stained with fluorochrome-conjugated mAbs for human leukocyte antigenDR isotype (HLA-DR) and CD86 (BD science, Erembodegem-Dorp, Belgium) or control Abs. Data was acquired on a Cytoflex cytometer (Beckman Coulter, Kraemer Boulevard Brea, CA, USA) and analyzed using FlowJo 10.3 software.

\section{Enzyme-Linked Immunosorbent Assay (ELISA)}

The concentration of interleukin 6 (IL-6) and tumor necrosis factor alpha (TNF- $\alpha$ ) was detected by an ELISA kits in accordance with the manufacturers' instructions (Biolegend, San Diego, CA, USA).

\section{Microarray Assay and Data Analysis}

A total of 10 RNA samples with different in situ HHLA2 staining were subjected to a microarray data study and was analyzed using R software (version 3.5.0). ${ }^{20}$ Genes with a fold-change $(\mathrm{FC})>2$ and all adjusted $P$-values $<0.05$ were considered to be statistically significant to acquire differentially expressed genes (DEGs). 


\section{Statistical Analysis}

The correlation analyses were performed using a $\chi^{2}$ test, and survival analysis was performed using the Kaplan-Meier method to calculate the survival curves of RFS and OS, and compared via a $\log$ rank test. Univariate and multivariate analyses were carried out using the Cox proportional hazard model. All statistical analyses were performed using SPSS V26.0 software (Chicago, IL, USA) and GraphPad Prism 8 software (La Jolla, California, USA). A $P<0.05$ was considered to be significant.

\section{Results}

\section{HHLA2 Expression in HCC Tissue}

To detect the pattern of HHLA2 expression in the HCC tumor tissues, 205 samples from patients with HCC who received a curative resection were examined. Among these patients, the majority were male $(184 / 205,89.3 \%), 92.2 \%$ (189/205) were positive for HBV infection, 50.7\% (104/ 205) had a large tumor size ( $>5 \mathrm{~cm}$ in diameter), $67.3 \%$ $(138 / 205)$ had a single tumor, and $17.6 \%$ (36/205) were observed for vascular invasion. According to the TNM staging system (7th Edition), 61.5\% (126/205) patients were defined as stages I and II. In addition, the histological grade of tumor differentiation was defined as I and II (116/ $205,56.6 \%)$, as well as III and IV (89/205, 43.4\%) according to the Edmondson grading system. The clinicopathological characteristics are summarized in Table 1.

HHLA2 staining was performed using IHC, and membranous HHLA2 expression was detected (Figure 1A). Positive membranous HHLA2 staining was primarily located on stromal cells. Of the 205 samples that were examined, positive HHLA2 staining was enriched in the peri-tumoral region of HCC tissues, compared with that in the non-tumor and tumor regions (Figure 1B). To identify the HHLA2-positive immune cell types, we performed immunofluorescence colocalization studies. As shown in Figure $1 \mathrm{C}$, the majority of HHLA2-expressing cells were $\mathrm{CD} 8^{+}$monocytes/macrophages (Mos/M $\varphi s$ ). No localization with $\mathrm{CD}^{+}$or $\mathrm{CD} 20^{+}$cells was observed. Collectively, these data suggest that HHLA2-expressing cells enriched in the peri-tumor region and were primarily $\mathrm{Mos} / \mathrm{M} \varphi \mathrm{s}$.

\section{Tumor-Activated Monocytes Upregulated HHLA2 Expression}

To confirm the level of HHLA2 expression on Mos/M $\varphi s$, monocytes isolated from healthy donors were examined. As shown in Figure 2A, under normal culture conditions,
Table I Patients' Characteristics

\begin{tabular}{|c|c|}
\hline Variable & No. of Patients (\%) \\
\hline No. of patients & $205(100)$ \\
\hline Age: median [range], y & $50[21-78]$ \\
\hline \multicolumn{2}{|l|}{ Gender } \\
\hline Male & I84 (89.3) \\
\hline Female & $21(10.7)$ \\
\hline \multicolumn{2}{|l|}{$\mathrm{HBsAg}$} \\
\hline Negative & $16(7.8)$ \\
\hline Positive & $189(92.2)$ \\
\hline \multicolumn{2}{|l|}{ Cirrhosis } \\
\hline Negative & $46(22.4)$ \\
\hline Positive & $159(77.6)$ \\
\hline \multicolumn{2}{|l|}{ ALT (U/L) } \\
\hline$<37$ & $88(42.9)$ \\
\hline$\geq 37$ & $117(57.1)$ \\
\hline \multicolumn{2}{|l|}{$\operatorname{AFP}(\mathrm{ng} / \mathrm{mL})$} \\
\hline$\leq 25$ & $65(31.7)$ \\
\hline$>25$ & $140(68.3)$ \\
\hline \multicolumn{2}{|l|}{ Tumor size $(\mathrm{cm})$} \\
\hline$>5$ & $104(50.7)$ \\
\hline$\leq 5$ & $101(49.3)$ \\
\hline \multicolumn{2}{|l|}{ Tumor number } \\
\hline Single & $138(67.3)$ \\
\hline Multiple & $67(32.7)$ \\
\hline \multicolumn{2}{|l|}{ Vascular invasion } \\
\hline Absent & $169(82.4)$ \\
\hline Present & $36(17.6)$ \\
\hline \multicolumn{2}{|l|}{ TNM stage } \\
\hline I+II & $126(6 \mid .5)$ \\
\hline III+IV & $79(38.5)$ \\
\hline \multicolumn{2}{|l|}{ Tumor differentiation } \\
\hline I-II & $116(56.6)$ \\
\hline III-IV & $89(43.3)$ \\
\hline
\end{tabular}

the level of HHLA2 transcription on $\mathrm{Mo} / \mathrm{M} \varphi$ was barely detectable; however, exposure to $10 \%$ TSNs from hepatoma (SK) cells induced rapid HHLA2 expression, peaking within $8 \mathrm{~h}$, and then rapidly declined. We also examined the activation status of TSN-exposed monocytes. Consistent with our previous reports, the level of HLA-DR and CD86 expression was significantly upregulated on monocytes following exposure to TSNs from SK cells for $24 \mathrm{~h}$, but was reduced on day 7 (Figure 2B). ${ }^{22,23}$ The accumulation of TNF- $\alpha$ and IL-6 
A

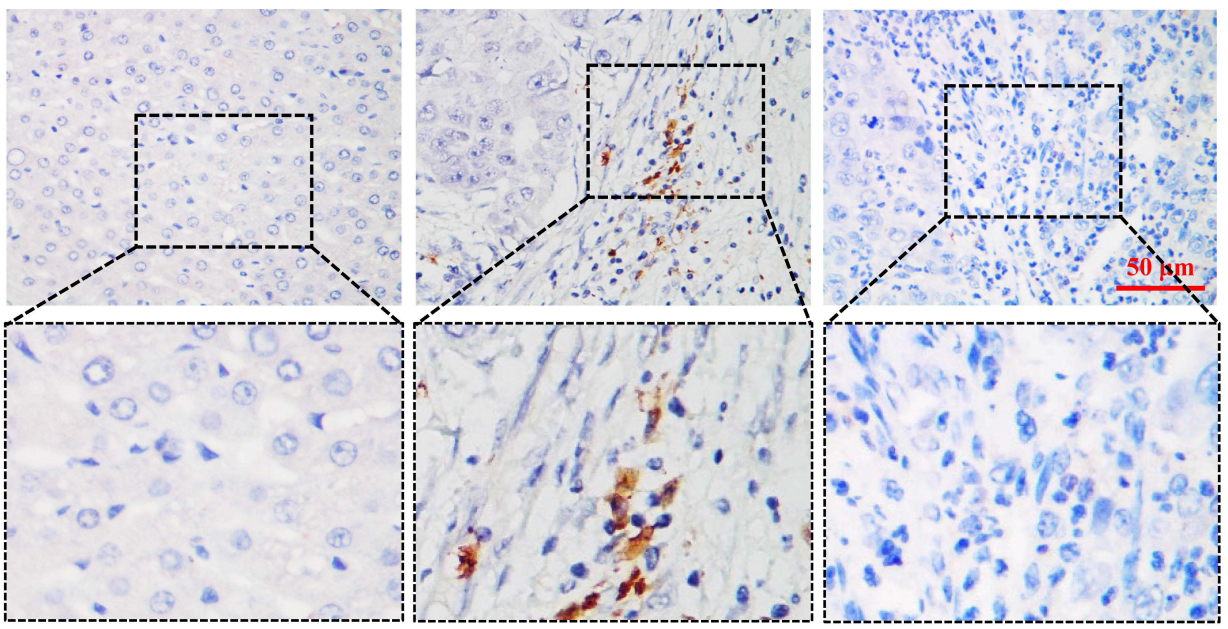

Tumor
B

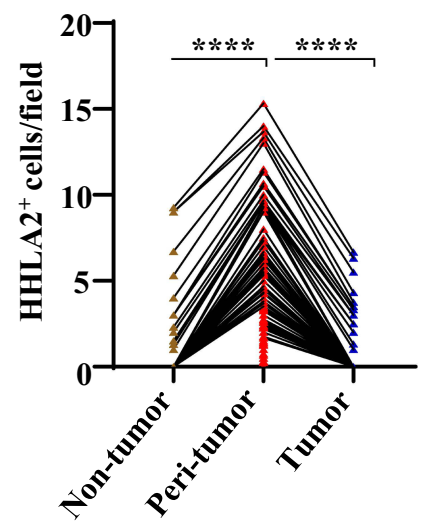

C

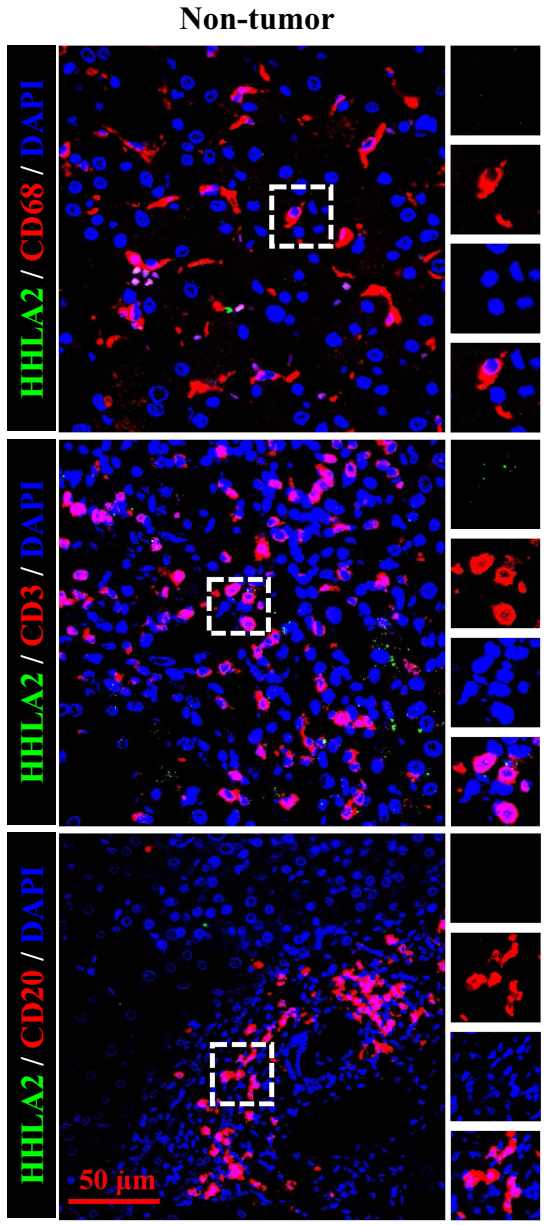

Peri-tumor
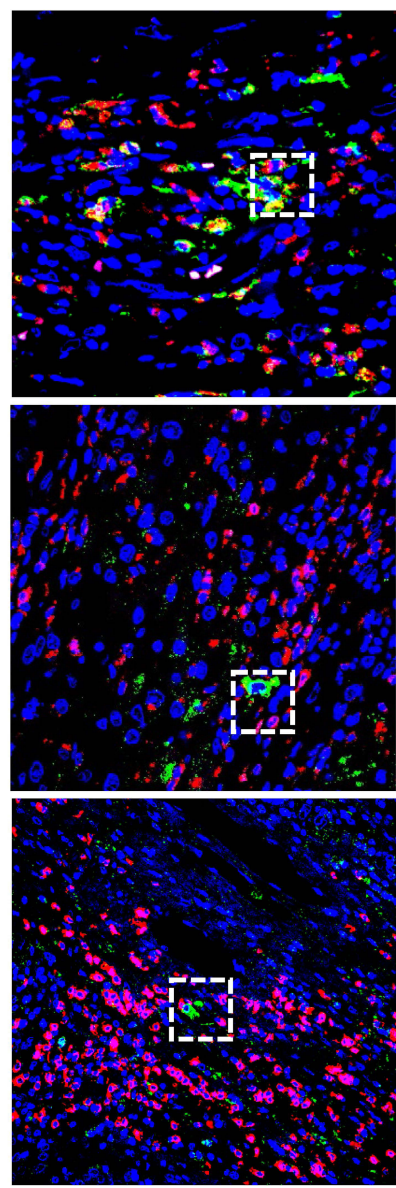
A

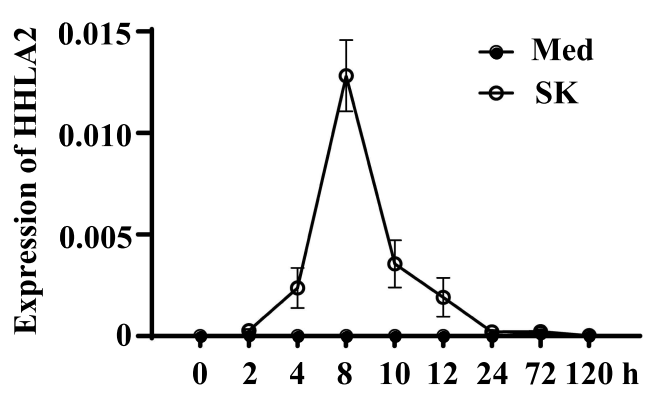

B

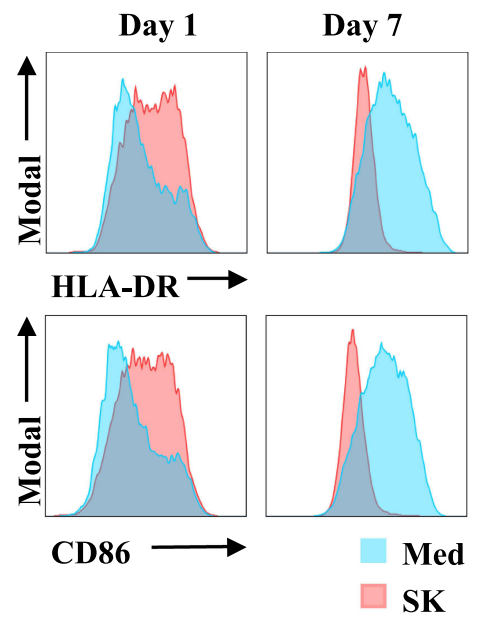

C

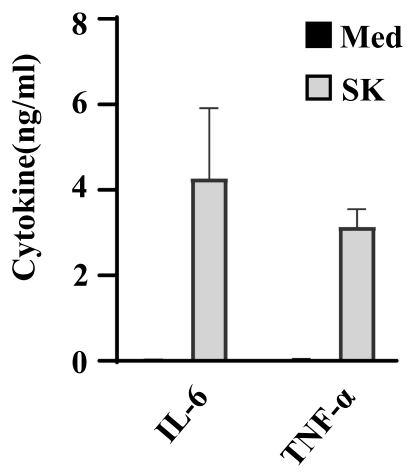

D

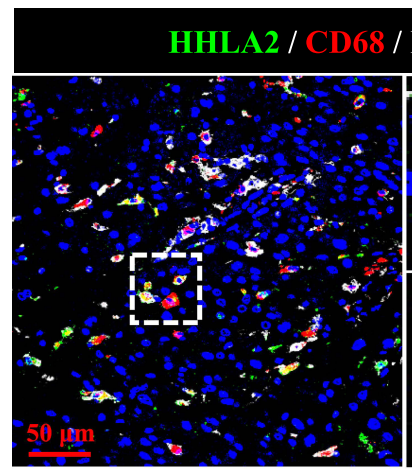

\section{HLA-DR /}

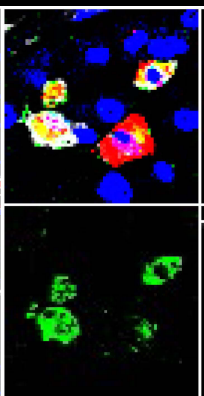

E

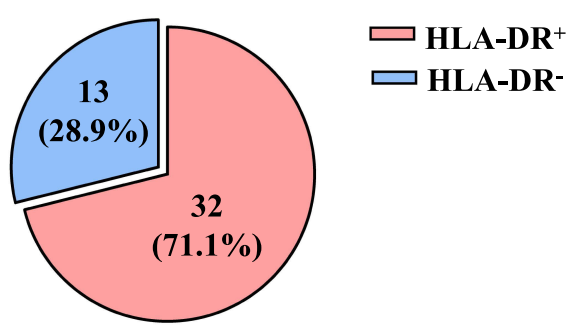

Figure 2 HHLA2 expression was associated with an activated state of monocytes and macrophages. (A) Monocytes were left untreated or cultured with SK supernatant for the indicated time points, after which the transcription levels of HHLA2 were determined by qPCR. (B and C) Monocytes were left untreated or cultured with SK supernatant for one or seven days. The levels of HLA-DR, CD86, IL-6, and TNF- $\alpha$ were determined by flow cytometry or an ELISA. (D) Multiplex immunofluorescence staining of HHLA2, CD68, and HLA-DR in HCC tissue (scale bar, $50 \mu \mathrm{m}$ ). HHLA2 staining is shown in green, CD68 staining is shown in red, HLA-DR staining is shown in white, and DAPI staining is shown in blue. (E) Manual enumeration was applied to calculate the number of CD68-, HHLA2-, and HLA-DR-positive cells, respectively. Pie charts show the percentage of the indicated cell populations.

was also found in the culture media of TSN-exposed monocytes for $24 \mathrm{~h}$ as determined by an ELISA (Figure 2C). These results indicate that tumor-activated monocytes upregulated HHLA2 expression.

Immunofluorescence staining of HHLA2, CD68, and HLA-DR was performed on HCC tissue sections. ${ }^{23}$ As shown in Figure 2D, $\mathrm{HHLA} 2^{+} \mathrm{CD} 68^{+}$cells expressed HLA-DR in the peri-tumor region of HCC. Statistics showed that $22.5 \%$ of $\mathrm{CD}^{+} 8^{+} \mathrm{Mo} / \mathrm{M} \varphi$ were positive for HHLA2, and $71.1 \%$ of these HHLA $2^{+} \mathrm{Mos} / \mathrm{M} \varphi s$ were HLA-DR-positive (Figure 2E). Taken together, these data suggest that HHLA2-expressing $\mathrm{Mos} / \mathrm{M} \varphi$ s exhibited an activated state in HCC tissues.

\section{HHLA2 Expression is Correlated with Disease Progression in HCC Patients}

To explore the impact of the HHLA2 expression on disease progression in patients with $\mathrm{HCC}$, we investigated the correlation between the HHLA2 expression and patient survival time. We divided the patients into two groups according to their HHLA2 expression in the peri-tumor region using the ROC curve. No significant correlation was identified between the level of HHLA2 expression and the patients' clinical characteristics (Table 2). The Kaplan-Meier survival analysis revealed a significant association between the presence of HHLA2-expressing cells and the patients' OS in the peri-tumor area $(P=0.008$; Figure 3A). The multivariate analysis revealed that HHLA2 expression in the peri-tumor area was an independent prognostic factor for $\mathrm{OS}(\mathrm{HR}=1.872,95 \% \mathrm{CI}=$ 1.236-2.835; $P=0.003$ ) (Table 3). These results reveal that the level of HHLA2 expression in the peri-tumor region negatively correlated with the prognosis of HCC patients. To further verify the prognostic role of HHLA2, we performed survival analysis in patients stratified by clinical features. As shown in Figure $3 \mathrm{~B}$ and $\mathrm{C}$, peri- 
Table 2 The Association of HHLA2 Expression with Clinicopathological Characteristics

\begin{tabular}{|c|c|c|c|c|}
\hline \multirow[t]{2}{*}{ Variable } & & \multicolumn{3}{|c|}{ HHLA2 } \\
\hline & & $\begin{array}{c}\text { Low } \\
\text { (Cases) }\end{array}$ & $\begin{array}{c}\text { High } \\
\text { (Cases) }\end{array}$ & $p$ \\
\hline Age (years) & $\begin{array}{l}\leq 50 \\
>50\end{array}$ & $\begin{array}{l}70 \\
67\end{array}$ & $\begin{array}{l}39 \\
29\end{array}$ & 0.398 \\
\hline Gender & $\begin{array}{l}\text { Male } \\
\text { Female }\end{array}$ & $\begin{array}{c}123 \\
14\end{array}$ & $\begin{array}{l}61 \\
7\end{array}$ & 0.981 \\
\hline $\mathrm{HBsAg}$ & $\begin{array}{l}\text { Negtive } \\
\text { Positive }\end{array}$ & $\begin{array}{c}10 \\
127\end{array}$ & $\begin{array}{c}5 \\
63\end{array}$ & 0.989 \\
\hline Cirrhosis & $\begin{array}{l}\text { Absdent } \\
\text { Present }\end{array}$ & $\begin{array}{c}33 \\
104\end{array}$ & $\begin{array}{l}13 \\
55\end{array}$ & 0.422 \\
\hline ALT (U/L) & $\begin{array}{l}<37 \\
\geq 37\end{array}$ & $\begin{array}{l}56 \\
81\end{array}$ & $\begin{array}{l}32 \\
36\end{array}$ & 0.4 \\
\hline $\operatorname{AFP}(\mathrm{ng} / \mathrm{mL})$ & $\begin{array}{l}\leq 25 \\
>25\end{array}$ & $\begin{array}{l}45 \\
92\end{array}$ & $\begin{array}{l}20 \\
48\end{array}$ & 0.619 \\
\hline Tumor size $(\mathrm{cm})$ & $\begin{array}{l}\leq 5 \\
>5\end{array}$ & $\begin{array}{l}69 \\
68\end{array}$ & $\begin{array}{l}32 \\
36\end{array}$ & 0.656 \\
\hline Tumor number & $\begin{array}{l}\text { Single } \\
\text { Multiple }\end{array}$ & $\begin{array}{l}91 \\
46\end{array}$ & $\begin{array}{l}47 \\
21\end{array}$ & 0.699 \\
\hline Vascular invasion & $\begin{array}{l}\text { Absent } \\
\text { Present }\end{array}$ & $\begin{array}{l}110 \\
27\end{array}$ & $\begin{array}{c}59 \\
9\end{array}$ & 0.251 \\
\hline TNM stage & $\begin{array}{c}I+I I \\
I I I+I V\end{array}$ & $\begin{array}{l}85 \\
52\end{array}$ & $\begin{array}{l}41 \\
27\end{array}$ & 0.809 \\
\hline $\begin{array}{l}\text { Tumor } \\
\text { differentiation }\end{array}$ & $\begin{array}{c}\text { I+II } \\
\text { III+IV }\end{array}$ & $\begin{array}{l}78 \\
59\end{array}$ & $\begin{array}{l}38 \\
30\end{array}$ & 0.886 \\
\hline
\end{tabular}

Abbreviations: AFP, alpha-fetoprotein; ALT, alanine aminotransferase; $\mathrm{HBsAg}$ hepatitis B surface antigen; TNM, tumor-lymph node metastasis.

tumoral HHLA2 was also associated OS in patients with small tumor, and patients with early stage of HCC. These results suggested that HHLA2 expression in the peri-tumor area could influence patients' survival independent of common clinical characteristics.

\section{Peri-Tumoral HHLA2 Expression is Associated with an Immune Inhibitory State in HCC Tumors}

Given that HHLA2 is an immune checkpoint molecule, we examined the relationship between HHLA2 expression and tumor-infiltrating immune cells. As shown in Figure 4A, high HHLA2 expression in the peri-tumor region was strongly correlated with an increased density of CD204 and $\mathrm{CD}_{11 \mathrm{~b}^{+}}$myeloid cells in the tumor regions. No significant correlation was observed between HHLA2 expression and the density of $\mathrm{CD} 163^{+}$or $\mathrm{CD} 15^{+}$cells.

To further characterize the relation between HHLA2 expression and local immune state, we analyzed the gene expression microarray data of 10 tumor samples with different in situ HHLA2 expression. The cut-off value for low and high HHLA2 expression in the peri-tumoral region was consistent with the results of the survival analysis. We compared peri-tumoral HHLA2 expression with immune-related genes to acquire differentially expressed genes. ${ }^{24} \mathrm{~A}$ total of 28 immune-related genes with the most significant expression were displayed (Figure 4B). The heatmap showed that the patients with high HHLA2 expression had lower CD3, CD4, neural cell adhesion molecule 1 (NCAM1, also called CD56), Granzyme B (GZMB), as well as higher T cell Ig and ITIM domain (TIGIT), CTLA4, and CD19 gene expression, indicating a potential immune inhibitory response in the tumor. Taken together, these data suggest that a high level of peri-tumoral HHLA2 expression is correlated with an inhibitory immune state in the HCC tumor nest.

\section{Impact of the PD-LI and HHLA2 Expression Pattern on the Disease Progression of HCC Patients}

Immune regulatory factors within the tumor microenvironment could induce both PD-L1 and HHLA2 expression. ${ }^{12,25}$ Therefore, we further analyzed the expression patterns of PD-L1 and HHLA2 in HCC tissues. As shown in Figure 4B, high CD274 gene expression (encoding PD-L1) was observed in tissues expressing low levels of HHLA2. Moreover, we performed in situ staining of PD-L1 in the peri-tumor region of HCC tissues (Figure 5A). The results showed that HHLA2 expression was negatively correlated with PD-L1 (Figure 5B), indicating the differential HHLA2 and PD-L1 expression patterns in HCC tissues.

We also examined immune cell infiltration and the impact of HHLA2/PD-L1 expression patterns on disease progression in HCC. Patients were divided into the following four groups based on the level of HHLA2 and PD-L1 expression in the peri-tumoral region: 1) low HHLA2 and PD-L1 expression (pattern 1: HHLA $2^{\text {Lo }}$-PD-L1 ${ }^{\text {Lo }}$; 2) high HHLA2 expression only (pattern 2: HHLA2 ${ }^{\mathrm{Hi}}-\mathrm{PD}-\mathrm{L} 1^{\mathrm{Lo}}$ ); 3) high PD-L1 expression only (pattern 3: HHLA2 ${ }^{\mathrm{Lo}}-\mathrm{PD}-\mathrm{L} 1^{\mathrm{Hi}}$ ); and 4) high HHLA2 and PD-L1 expression (pattern 4: HHLA2 ${ }^{\mathrm{Hi}}$-PD 
A All patients

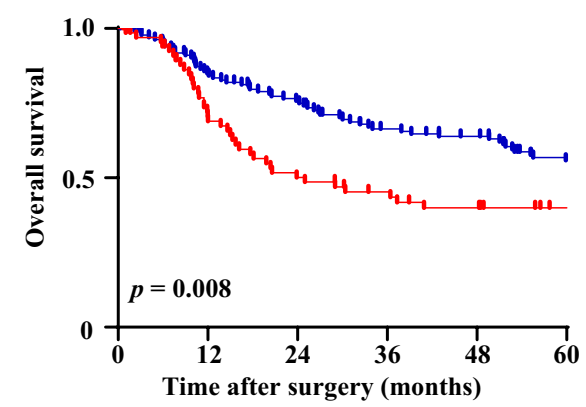

Number at risk

$\begin{array}{rlrrrrr}\text { High } & 68 & 45 & 32 & 26 & 22 & 16 \\ \text { Low } & 137 & 112 & 98 & 82 & 76 & 59\end{array}$

B Tumor size $\leq \mathbf{5} \mathrm{cm}$

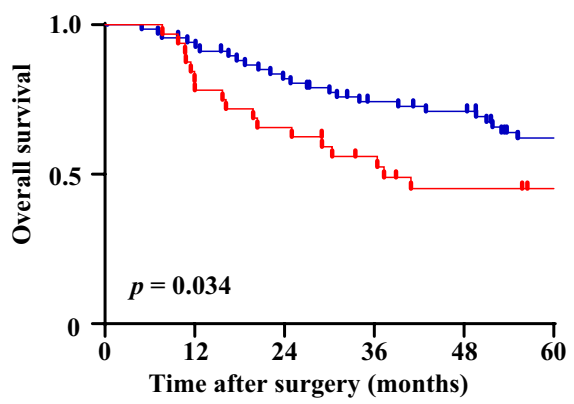

Number at risk

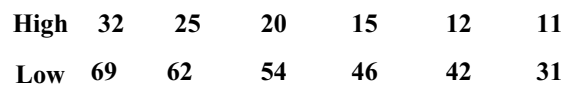

\section{C $\quad$ TNM $=$ I+II}

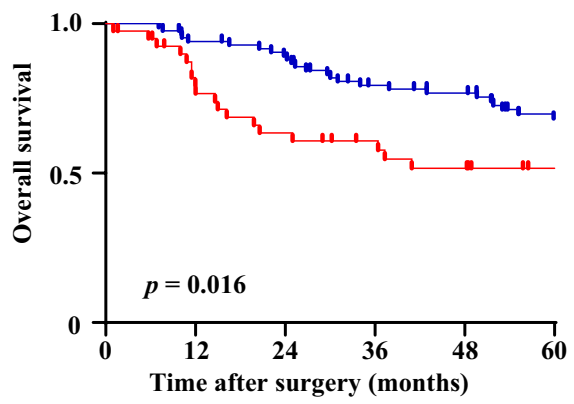

Number at risk

$\begin{array}{ccccccc}\text { High } & 41 & 29 & 23 & 19 & 16 & 11 \\ \text { Low } & 85 & 79 & 73 & 62 & 57 & 45\end{array}$

HHLA2

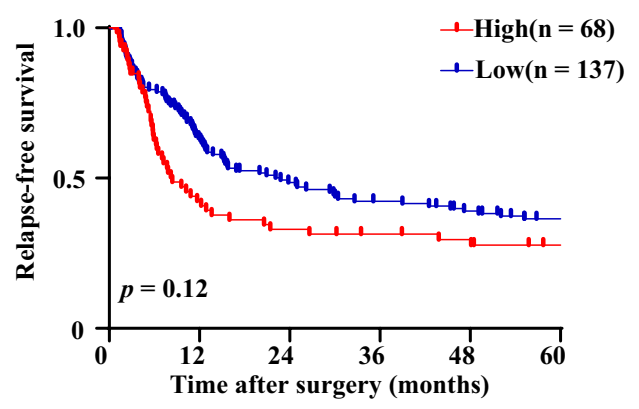

Number at risk

$\begin{array}{lllllll}\text { High } & 68 & 27 & 21 & 18 & 16 & 12 \\ \text { Low } & 137 & 82 & 62 & 53 & 47 & 37\end{array}$

HHLA2

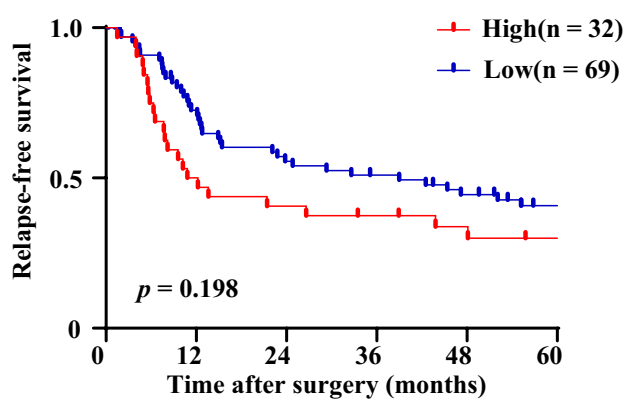

Number at risk
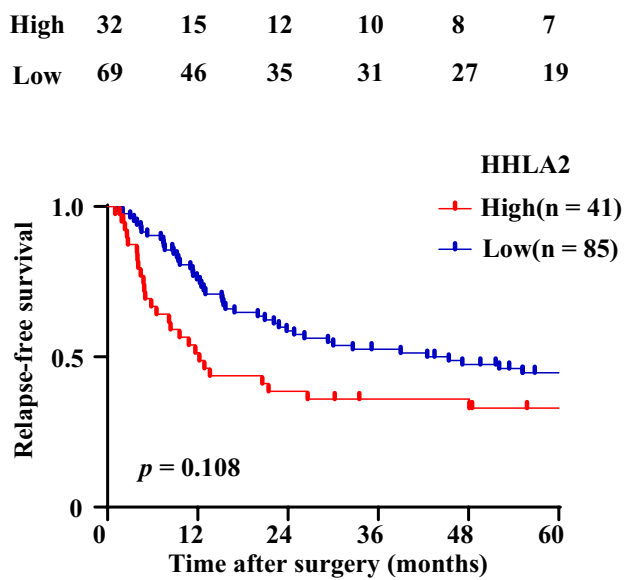

Number at risk

$\begin{array}{lllllll}\text { High } & 41 & 19 & 14 & 12 & 11 & 8 \\ \text { Low } & 85 & 61 & 47 & 41 & 36 & 28\end{array}$

Figure 3 The impact of HHLA2 expression on the OS and RFS of HCC patients. (A) The impact of HHLA2 expression on OS and RFS were calculated using the KaplanMeier method and analyzed using a Log rank test. (B) The impact of HHLA2 expression on OS and RFS were calculated in patients with tumor size $\leq 5 \mathrm{~cm}$. (C) The impact of HHLA2 expression on OS and RFS were calculated in patients with TNM I and II.

$-\mathrm{L} 1^{\mathrm{Hi}}$ ). $\mathrm{CD} 163^{+}$and $\mathrm{CD} 204^{+}$cells accumulated in tumor tissues with high HHLA2 and PD-L1 expression (Figure 5C). The Kaplan-Meier survival analysis showed that patients exhibiting high HHLA2 and PD-
L1 expression in the peri-tumor region displayed the worst clinical outcomes (Figure 5D). Taken together, these results reveal different HHLA2 and PD-L1 expression patterns in HCC, and high HHLA2 and PD-L1 
Table 3 Univariate and Multivariate Analysis of Factors Associated with Patients' Overall Survival

\begin{tabular}{|c|c|c|c|c|c|c|}
\hline \multirow[t]{2}{*}{ Variables } & \multicolumn{3}{|c|}{ Univariate } & \multicolumn{3}{|c|}{ Multivariate } \\
\hline & HR & $95 \% \mathrm{Cl}$ & $p^{a}$ & HR & $95 \% \mathrm{Cl}$ & $p^{a}$ \\
\hline Age,yr $(\leq 50 />50)$ & 0.981 & $0.660-1.459$ & 0.925 & & & \\
\hline Gender (male/female) & 1.285 & $0.716-2.304$ & 0.401 & & & \\
\hline HBsAg (positive/negative) & 0.966 & $0.448-2.085$ & 0.930 & & & \\
\hline Cirrhosis (present/absent) & 1.264 & $0.740-2.160$ & 0.391 & & & \\
\hline ALT, U/L $(\geq 37 /<37)$ & 1.546 & $1.019-2.344$ & 0.040 & 1.576 & $1.026-2.421$ & 0.038 \\
\hline AFP, ng/mL $(>25 / \leq 25)$ & 1.735 & $|.093-2.75|$ & 0.019 & & & na \\
\hline Tumor size, cm $(>5 / \leq 5)$ & 1.179 & $0.748-1.8529$ & 0.478 & & & \\
\hline Tumor number (multiple/single) & 1.648 & I.105-2.457 & 0.014 & & & na \\
\hline Vascular invasion (present/absent) & 4.849 & $3.054-7.697$ & $<0.001$ & 3.451 & $2.068-5.760$ & $<0.001$ \\
\hline TNM stage $(I I I+\mid \mathrm{V} / I+I I)$ & 2.725 & $1.829-4.060$ & $<0.001$ & 1.978 & I.272-3.076 & 0.002 \\
\hline Differentiation $(\mathrm{III}+\mathrm{IV} / \mathrm{I}+\mathrm{II})$ & 1.089 & $0.731-1.622$ & 0.646 & & & \\
\hline HHLA2 (high/low) & 1.677 & $1.117-2.518$ & 0.013 & 1.872 & $1.236-2.835$ & 0.003 \\
\hline
\end{tabular}

Notes: Cox proportional hazards regression model; variables that were associated with overall survival in the univariate analysis were adopted as covariates in the multivariate analysis and were entered into the equation using the forward likelihood ratio method. ${ }^{a} A$ Italic values indicate significance of $p$ value $(p<0.05)$.

Abbreviations: AFP, alpha-fetoprotein; ALT, alanine aminotransferase; HBsAg, hepatitis B surface antigen; TNM, tumor-lymph node metastasis; Cl, confidence interval; na, not applicable.

expression could identify the patients with the worst survival.

\section{Discussion}

The present study explored the level of protein expression and clinical significance of $\mathrm{B} 7$ the family member, HHLA2, in human HCC. We found HHLA2 was expressed primarily on $\mathrm{Mos} / \mathrm{M} \varphi$ s enriched in the peritumoral region. A high level of HHLA2 expression was negatively correlated with prognosis in HCC patients, and indicated an immune inhibitory state in the tumor nest. In addition, patients with high HHLA2 and PD-L1 expression in the peri-tumor region displayed worst clinical outcomes.

HHLA2 expression has been reported to be expressed in various cancers. For example, in triple-negative breast cancer, Janakiram et al found that patients with high HHLA2 expression have higher lymph node metastasis and later clinical stages. ${ }^{10}$ However, there remain some controversial reports about the relationship between HHLA2 expression and the prognosis of HCC. Masataka et al found that the level of HHLA2 mRNA transcription in the peripheral blood of gastric cancer patients was significantly lower than that of normal people. Patients with low HHLA2 mRNA expression in the peripheral blood tend to have a later clinical stage and shorter fiveyear survival period, which suggests that low HHLA2 expression represents a poor prognosis in gastric cancer. $^{26}$ In contrast, the study by Wei et al found that HHLA2 mRNA and protein expression was significantly increased in gastric cancer tissues, which tended to be correlated with a later clinical stage and shorter OS time, indicating that high HHLA2 expression indicates a poor patient prognosis. ${ }^{27}$ The contradictory results of these studies may be caused by a weak correlation between HHLA2 mRNA expression in the peripheral blood and in situ protein expression in the tumor. To date, the level of HHLA2 expression in HCC tissues and associated clinical significance has not been reported. Therefore, in this study, we performed IHC on tumor tissues isolated from 205 HCC patients and found that HHLA2 expression was primarily detected on stromal cells in the peri-tumor area of HCC tumors, and was negatively correlated with patients' prognosis. Further analysis in patients stratified by clinical features showed that, peri-tumoral HHLA2 was also associated OS in patients with small tumor, and patients with early stage of HCC. These results suggested that HHLA2 expression in the peri-tumor area could influence patients' survival independent of common clinical characteristics.

The relationship between HHLA2 expression and immune cell infiltration has been reported in various cancers. Most studies have focused on lymphocytes and found negative correlations between HHLA2 expression and $\mathrm{T}$ cell infiltration (eg, cholangiocarcinoma, colorectal carcinoma, and clear cell renal carcinoma). ${ }^{17,19,28,29}$ However, some research has also reported a positive association between HHLA2 expression with high TIL density (eg, lung cancer) ${ }^{30}$ In our study, we found that the 
A

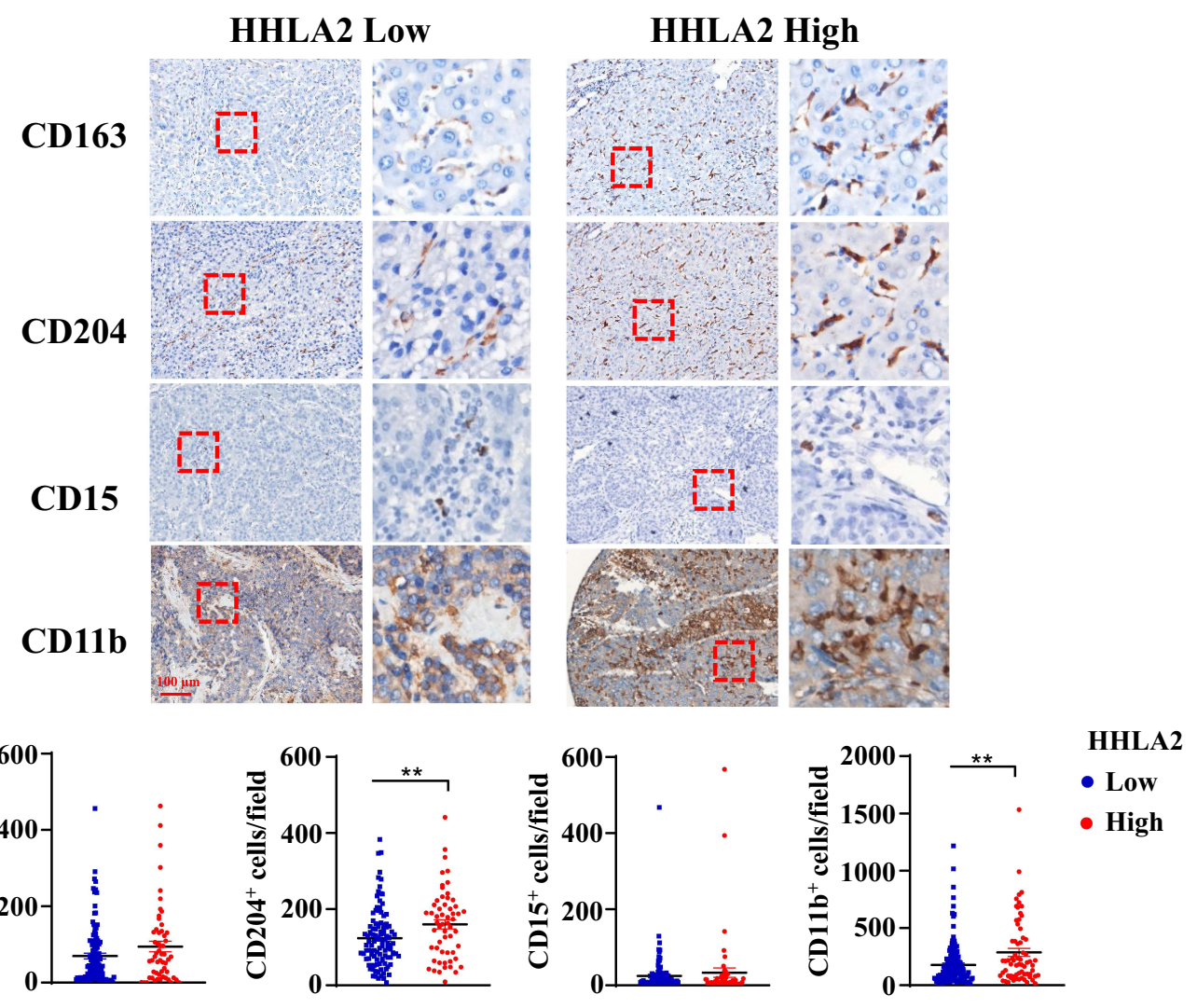

\section{B}

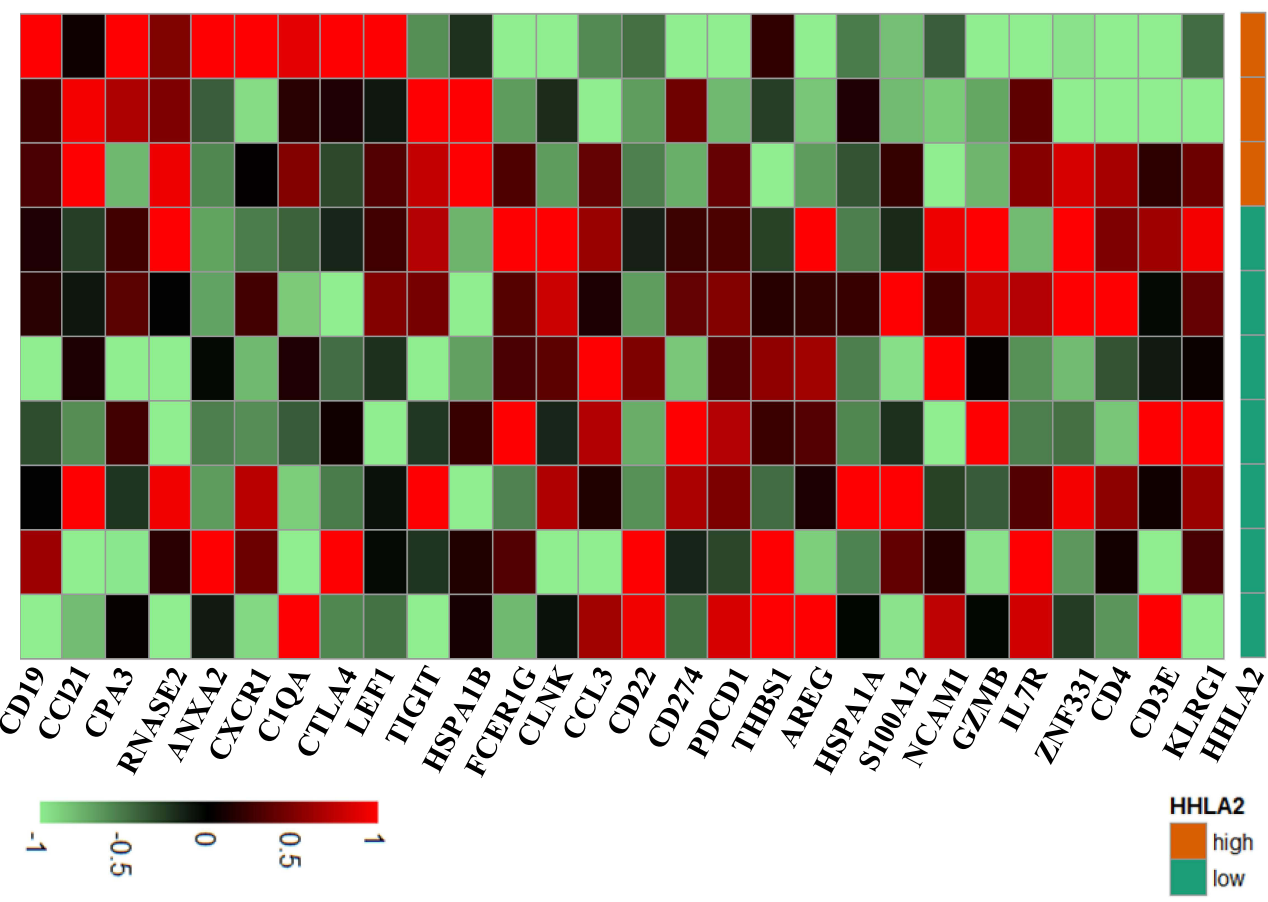

Figure 4 HHLA2 expression indicated an immune inhibitory microenvironment in the tumor nest of HCC. (A) Representative images of CDI63, CD 204, CDI5, and CDI lb by IHC staining in HCC tissue, and the correlation between HHLA2 expression in the peri-tumor region and myeloid cell infiltration in the tumor nest of HCC (scale bar: $100 \mu \mathrm{m}$ ). $* * P<0.01$. (B) A heatmap shows the relationship between the expression of immune-related genes and HHLA2 in the peri-tumor region of HCC. Gene expression was normalized, and lima package was employed to acquire the differential gene expression. 
A

PD-L1

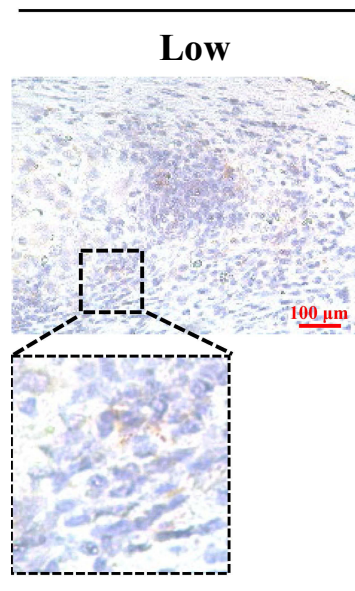

C

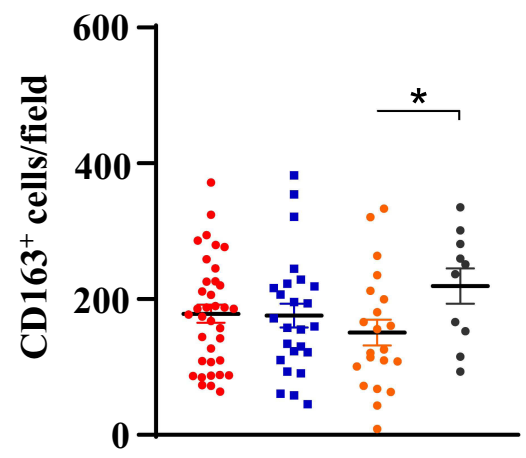

B

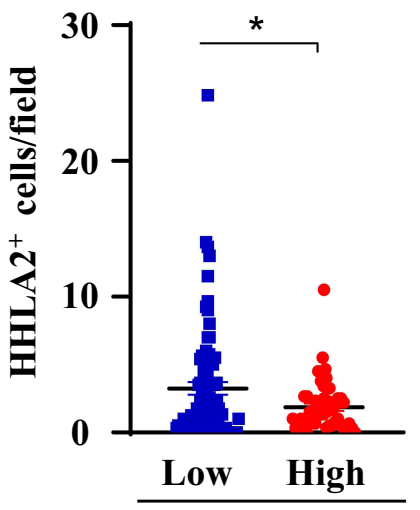

PD-L1
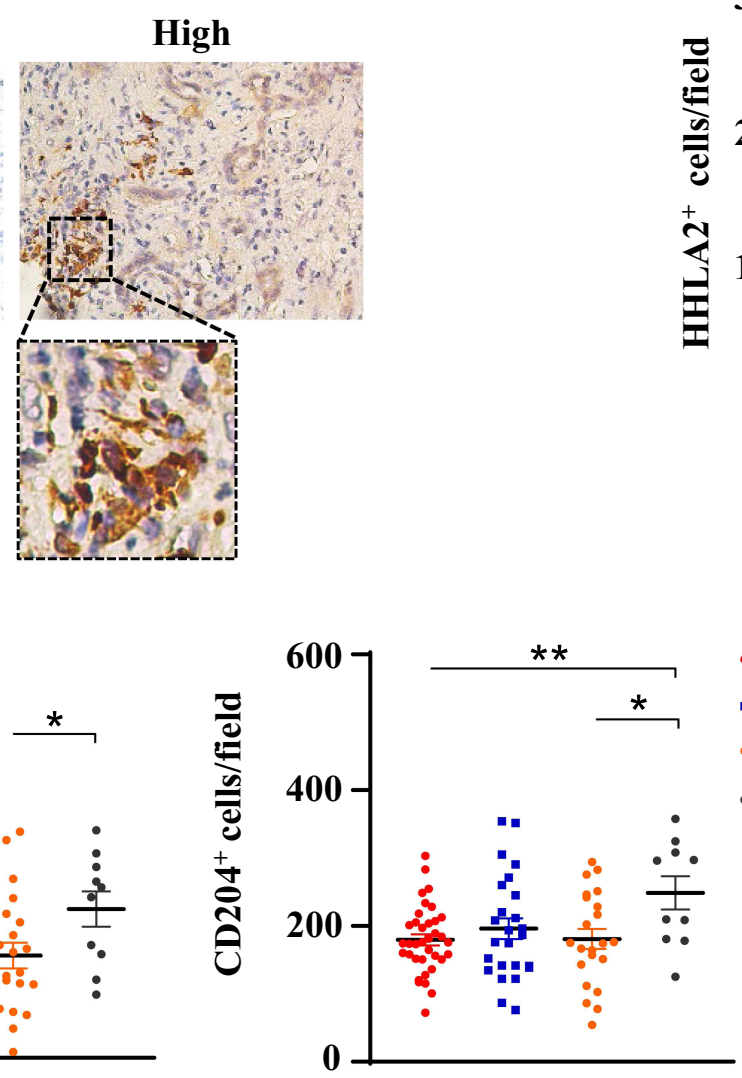

- HHLA2 ${ }^{\mathrm{Lo}}$ PD-L1 ${ }^{\mathrm{Lo}}$

- HHLA2 ${ }^{\mathrm{Lo}}$ PD-L1 ${ }^{\mathrm{Hi}}$

- HHLA2 ${ }^{\text {Hi PD-L1 }}{ }^{\text {Lo }}$

- HHLA2 $^{\mathrm{Hi}}$ PD-L1 ${ }^{\mathrm{Hi}}$

0

$\mathbf{D}$
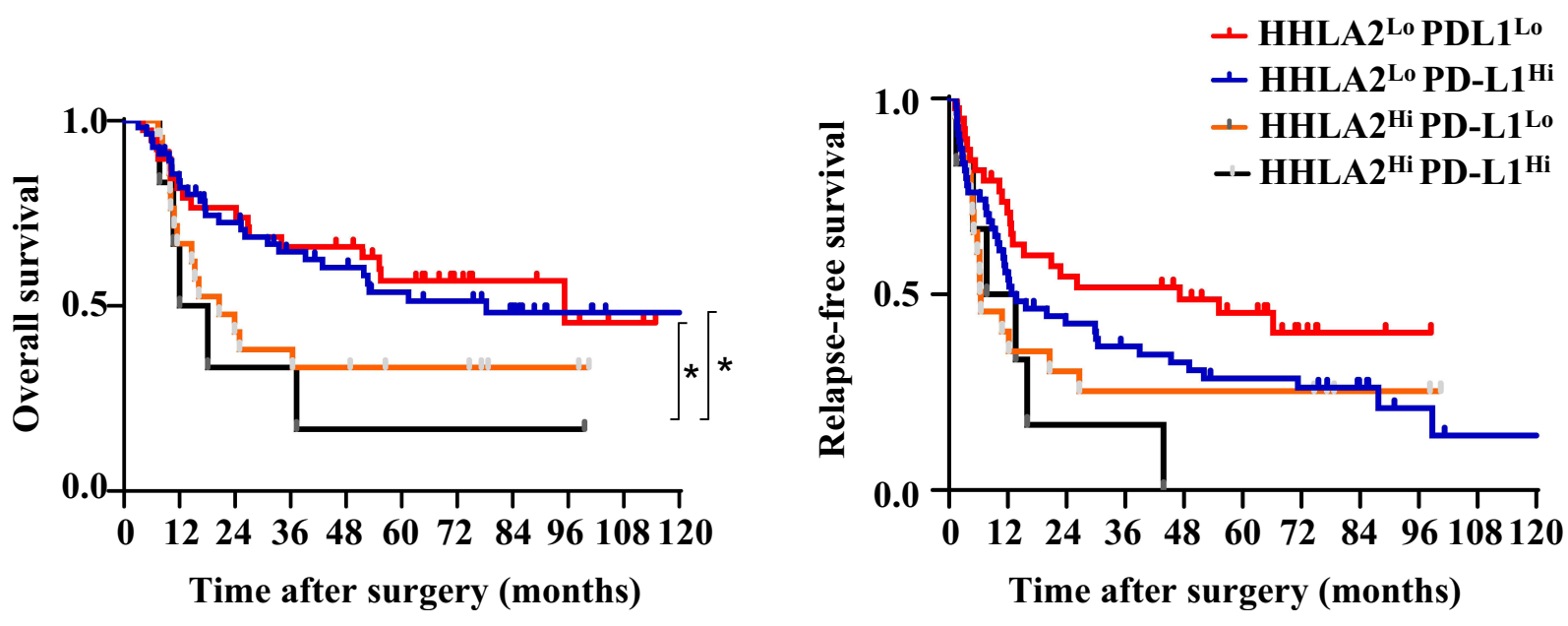

Figure 5 Different expression patterns of HHLA2 and PD-LI in HCC. (A) Representative images of PD-LI expression in the peri-tumor region of HCC tissue (scale bar: $100 \mu \mathrm{m}$ ). (B) The correlation between HHLA2 and PD-LI expression in the peri-tumor region of HCC. (C) The relationship between HHLA2/PD-LI expression patterns, as well as the infiltration of $\mathrm{CDI}_{63}{ }^{+}$and $\mathrm{CD} 204^{+}$cells. (D) Kaplan-Meier curves of HHLA2/PD-LI expression patterns in HCC patients. $* P<0.05$ and $* * P<0.01$. 
presence of HHLA2 was associated with high densities of $\mathrm{CD}_{20} 4^{+}$and $\mathrm{CD} 1 \mathrm{~b}^{+}$cellular infiltrations in HCC tumors. The high densities of these myeloid cell infiltrates often lead to tumor progression, ${ }^{21,31}$ and thus, might explain the negative influence of HHLA2 expression on the survival of HCC patients.

Previous studies have reported that the function of HHLA2 interacts with T cells; however, the role of HHLA2 in activating $\mathrm{T}$ cells remains controversial. The study by Zhao et al demonstrated that HHLA2 could inhibit human $\mathrm{CD}^{+}$and $\mathrm{CD}^{+} \mathrm{T}$ cell functionality through an unknown receptor located on the $\mathrm{T}$ cell membrane. ${ }^{11} \mathrm{Zhu}$ et al reported that HHLA2 could co-stimulate human T-cell growth and cytokine production through interacting with $\mathrm{CD} 28 \mathrm{H}^{12}$ Moreover, Rieder et al discovered that HHLA2 was insufficient to evaluate the proliferation and cytokine production of $\mathrm{T}$ cells stimulated with OKT3 (the anti-CD3 monoclonal antibody), which may result from the differential effects of various $\alpha$ CD3 clones on T cell function. ${ }^{13}$ Recent studies demonstrated inhibitory effects of HHLA2 on T cells and NK cells, when interacts with the inhibitory receptor KIRR3DL3, ${ }^{14}$ suggesting HHLA2 as a potential immunotherapeutic target for cancer. In this study, we focused on the in situ distribution of HHLA2 in HCC tissue, and identified a high expression of HHLA2 on activated monocytes/macrophages in the peri-tumor region of HCC. The high expression of peri-tumoral HHLA2 correlated with an inhibitory immune state in the HCC tumor nest, and poor clinical outcomes of patients, indicating an immunosuppressive role of HHLA2 in HCC. Further understanding of the precise mechanism that HHLA2 modulates anti-tumor immunity in HCC deserves future investigation, such as the expression of its receptors during tumor progression.

Previous studies in lung cancer have reported that positive HHLA2 and PD-L1 expression were both relatively low; however, patients with negative expression of PD-L1 exhibited high HHLA2 expression. ${ }^{32}$ Consistently, we found that the level of HHLA2 expression was negatively correlated with PD-L1 in the peri-tumor region of $\mathrm{HCC}$, indicating their expression might be differently regulated in the tissue. Despite the different expression patterns, high HHLA2 expression also indicated a suppressive tumor microenvironment. These results suggest that targeting HHLA2 may serve as a potential complement for anti-PD-L1 therapy. In conclusion, this study furthers our understanding of the expression and clinical relevance of the immune checkpoint family. Therefore, HHLA2 may serve as a potential target for tumor immunotherapy.

\section{Funding}

This work was supported by project grants from the National Key R\&D Program of China (2017YFA0505803), the National Natural Science Foundation of China (81772536), and the Science and Information Technology of Guangzhou (201904020040).

\section{Disclosure}

The authors report no conflicts of interest in this work.

\section{References}

1. Villanueva A, Longo DL. Hepatocellular carcinoma. $N$ Engl J Med. 2019;380(15):1450-1462. doi:10.1056/NEJMra1713263

2. Forner A, Reig M, Bruix J. Hepatocellular carcinoma. Lancet. 2018;391(10127):1301-1314. doi:10.1016/S0140-6736(18)30010-2

3. Pitt JM, Vétizou M, Daillère R, et al. Resistance mechanisms to immune-checkpoint blockade in cancer: tumor-intrinsic and extrinsic factors. Immunity. 2016;44(6):1255-1269. doi:10.1016/j. immuni.2016.06.001

4. Zhang Q, Vignali DAA. Co-stimulatory and co-inhibitory pathways in autoimmunity. Immunity. 2016;44(5):1034-1051. doi:10.1016/j. immuni.2016.04.017

5. Pardoll DM. The blockade of immune checkpoints in cancer immunotherapy. Nat Rev Cancer. 2012;12(4):252-264. doi:10.1038/ $\operatorname{nrc} 3239$

6. Janakiram M, Shah UA, Liu W, Zhao A, Schoenberg MP, Zang X. The third group of the B7-CD28 immune checkpoint family: HHLA2, TMIGD2, B7x, and B7-H3. Immunol Rev. 2017;276 (1):26-39. doi:10.1111/imr.12521

7. Qin S, Xu L, Yi M, Yu S, Wu K, Luo S. Novel immune checkpoint targets: moving beyond PD-1 and CTLA-4. Mol Cancer. 2019;18 (1):155. doi:10.1186/s12943-019-1091-2

8. Mager DL, Hunter DG, Schertzer M, Freeman JD. Endogenous retroviruses provide the primary polyadenylation signal for two new human genes (HHLA2 and HHLA3). Genomics. 1999;59 (3):255-263. doi:10.1006/geno.1999.5877

9. Janakiram M, Chinai JM, Zhao A, Sparano JA, Zang X. HHLA2 and TMIGD2: new immunotherapeutic targets of the B7 and CD28 families. Oncoimmunology. 2015;4(8):e1026534. doi:10.1080/ 2162402X.2015.1026534

10. Janakiram M, Chinai JM, Fineberg S, et al. Expression, clinical significance, and receptor identification of the newest b7 family member HHLA2 protein. Clin Cancer Res. 2015;21(10):2359-2366. doi:10.1158/1078-0432.CCR-14-1495

11. Zhao R, Chinai JM, Buhl S, et al. HHLA2 is a member of the B7 family and inhibits human CD4 and CD8 T-cell function. Proc Natl Acad Sci USA. 2013;110(24):9879-9884. doi:10.1073/ pnas. 1303524110

12. Zhu Y, Yao S, Iliopoulou BP, et al. B7-H5 costimulates human T cells via CD28H. Nat Commun. 2013;4(1):2043. doi:10.1038/ncomms3043

13. Rieder SA, Wang J, White N, et al. B7-H7 (HHLA2) inhibits T-cell activation and proliferation in the presence of TCR and CD28 signaling. Cell Mol Immunol. 2020;18(6):1503-11.

14. Bhatt RS, Berjis A, Konge JC, et al. KIR3DL3 is an inhibitory receptor for HHLA2 that mediates an alternative immunoinhibitory pathway to PD1. Cancer Immunol Res. 2021;9(2):156-169. doi:10.1158/2326-6066.CIR-20-0315

15. Yan H, Qiu W, Koehne de Gonzalez AK, et al. HHLA2 is a novel immune checkpoint protein in pancreatic ductal adenocarcinoma and predicts post-surgical survival. Cancer Lett. 2019;442:333-340. doi:10.1016/j.canlet.2018.11.007 
16. Boor PPC, Sideras K, Biermann K, et al. HHLA2 is expressed in pancreatic and ampullary cancers and increased expression is associated with better post-surgical prognosis. Br J Cancer. 2020;122 (8):1211-1218. doi:10.1038/s41416-020-0755-4

17. Jing C-Y, Fu Y-P, Yi Y, et al. HHLA2 in intrahepatic cholangiocarcinoma: an immune checkpoint with prognostic significance and wider expression compared with PD-L1. J Immunother Cancer. 2019;7 (1):77. doi:10.1186/s40425-019-0554-8

18. Chen L, Zhu D, Feng J, et al. Overexpression of HHLA2 in human clear cell renal cell carcinoma is significantly associated with poor survival of the patients. Cancer Cell Int. 2019;19(1):101. doi:10.1186/s12935-019-0813-2

19. Zhou Q-H, Li K-W, Chen X, et al. HHLA2 and PD-L1 co-expression predicts poor prognosis in patients with clear cell renal cell carcinoma. J Immunother Cancer. 2020;8(1):e000157. doi:10.1136/ jitc-2019-000157

20. Liu C-Q, Xu J, Zhou Z-G, et al. Expression patterns of programmed death ligand 1 correlate with different microenvironments and patient prognosis in hepatocellular carcinoma. $B r J$ Cancer. 2018;119 (1):80-88. doi:10.1038/s41416-018-0144-4

21. Wu C, Lin J, Weng $\mathrm{Y}$, et al. Myeloid signature reveals immune contexture and predicts the prognosis of hepatocellular carcinoma. $J$ Clin Invest. 2020;130(9):4679-4693. doi:10.1172/JCI135048

22. Kuang D-M, Wu Y, Chen N, Cheng J, Zhuang S-M, Zheng L. Tumorderived hyaluronan induces formation of immunosuppressive macrophages through transient early activation of monocytes. Blood. 2007;110(2):587-595. doi:10.1182/blood-2007-01-068031

23. Kuang D-M, Zhao Q, Peng C, et al. Activated monocytes in peritumoral stroma of hepatocellular carcinoma foster immune privilege and disease progression through PD-L1. J Exp Med. 2009;206 (6):1327-1337. doi:10.1084/jem.20082173

24. Zheng C, Zheng L, Yoo J-K, et al. Landscape of infiltrating T cells in liver cancer revealed by single-cell sequencing. Cell. 2017;169 (7):1342-1356.e16. doi:10.1016/j.cell.2017.05.035
25. Jiang X, Wang J, Deng X, et al. Role of the tumor microenvironment in PD-L1/PD-1-mediated tumor immune escape. Mol Cancer. 2019;18(1):10. doi:10.1186/s12943-018-0928-4

26. Masataka S, Arigami T, Yanagita S, et al. The association of human endogenous retrovirus-H long terminal repeat-associating protein 2 (HHLA2) expression with gastric cancer prognosis. Oncotarget. 2018;9(31):22069-22078. doi:10.18632/oncotarget.25179

27. Wei L, Tang L, Chang H, Huo S, Li Y. HHLA2 overexpression is a novel biomarker of malignant status and poor prognosis in gastric cancer. Hum Cell. 2020;33(1):116-122. doi:10.1007/s13577-01900280-2

28. Zhu Z, Dong W. Overexpression of HHLA2, a member of the B7 family, is associated with worse survival in human colorectal carcinoma. Onco Targets Ther. 2018;11:1563-1570. doi:10.2147/ OTT.S160493

29. Chen $\mathrm{D}$, Chen $\mathrm{W}, \mathrm{Xu} \mathrm{Y}$, et al. Upregulated immune checkpoint HHLA2 in clear cell renal cell carcinoma: a novel prognostic biomarker and potential therapeutic target. J Med Genet. 2019;56 (1):43-49. doi:10.1136/jmedgenet-2018-105454

30. Cheng H, Janakiram M, Borczuk A, et al. HHLA2, a new immune checkpoint member of the B7 family, is widely expressed in human lung cancer and associated with EGFR mutational status. Clin Cancer Res. 2017;23(3):825-832. doi:10.1158/1078-0432.CCR-15-3071

31. Li J-Q, Yu X-J, Wang Y-C, et al. Distinct patterns and prognostic values of tumor-infiltrating macrophages in hepatocellular carcinoma and gastric cancer. J Transl Med. 2017;15(1):37. doi:10.1186/s12967017-1139-2

32. Cheng $\mathrm{H}$, Borczuk A, Janakiram M, et al. Wide expression and significance of alternative immune checkpoint molecules, B7x and HHLA2, in PD-L1-negative human lung cancers. Clin Cancer Res. 2018;24(8):1954-1964. doi:10.1158/1078-0432.CCR-17-2924

\section{Publish your work in this journal}

Biologics: Targets and Therapy is an international, peer-reviewed journal focusing on the patho-physiological rationale for and clinical application of Biologic agents in the management of autoimmune diseases, cancers or other pathologies where a molecular target can be identified. This journal is indexed on PubMed Central, CAS, EMBase,
Scopus and the Elsevier Bibliographic databases. The manuscript management system is completely online and includes a very quick and fair peer-review system, which is all easy to use. Visit $\mathrm{http}: / / \mathrm{www}$.dovepress.com/testimonials.php to read real quotes from published authors. 